Patricia Ex, Cornelia Henschke

\title{
Changing payment instruments and the utilisation of new medical technologies
}

Journal article | Accepted manuscript (Postprint)

This version is available at https://doi.org/10.14279/depositonce-8663

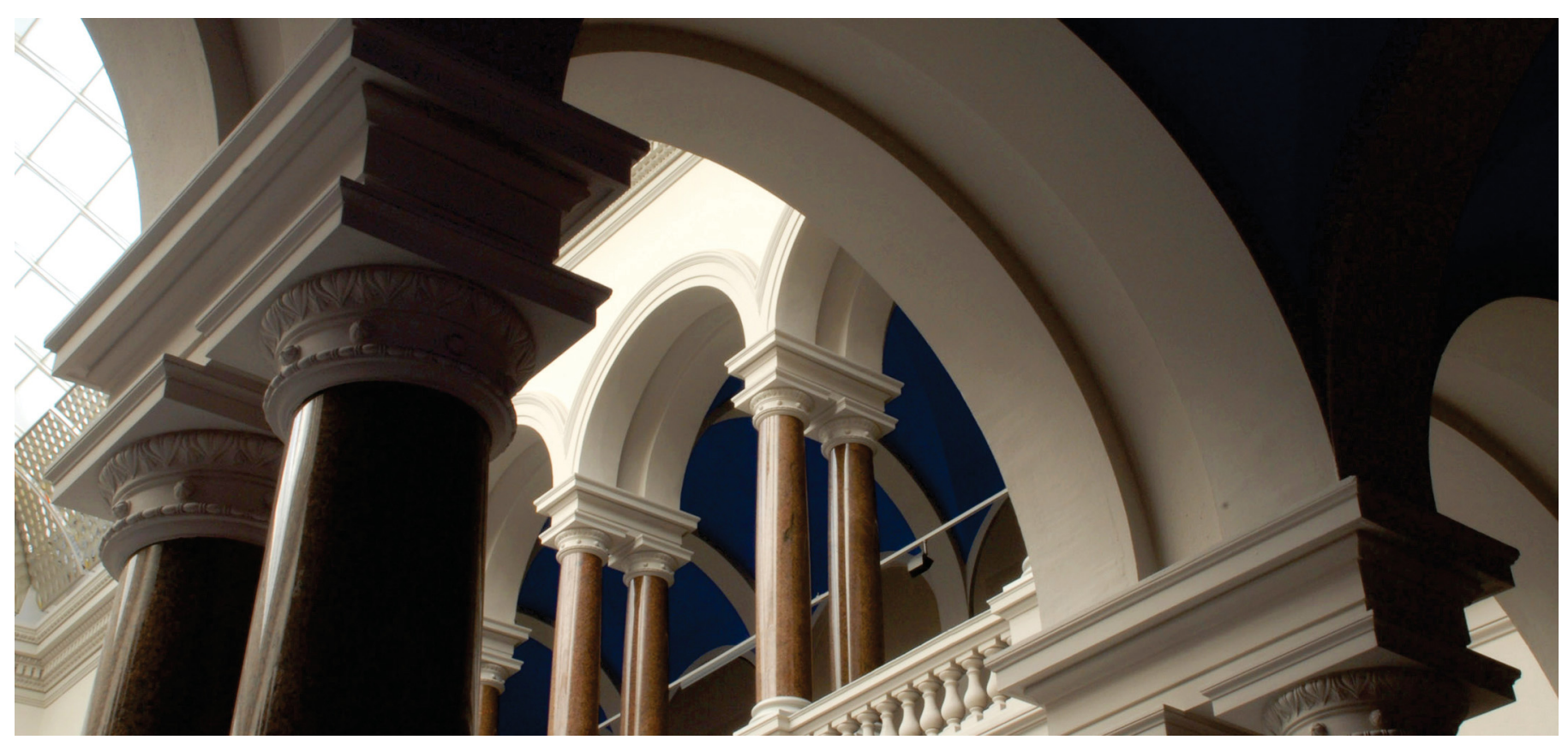

This is a post-peer-review, pre-copyedit version of an article published in The European Journal of Health Economics. The final authenticated version is available online at: http://dx.doi.org/10.1007/s10198-019-01056-z

Ex, P., \& Henschke, C. (2019). Changing payment instruments and the utilisation of new medical technologies. The European Journal of Health Economics. https://doi.org/10.1007/s10198-019-01056-z 


\title{
Changing payment instruments and
}

\section{the utilisation of new medical technologies}

\begin{abstract}
This paper empirically investigates the impact of additional reimbursement instruments on the diffusion of new technologies in inpatient care. Using 2010-2014 German panel data on hospital level for every patient undergoing coronary angioplasty, this study examines the utilisation of drug-eluting balloon catheters (DEB) over time while additional payment instruments changed. Hypothesising that the utilisation of DEB increased abruptly when a new reimbursement instrument came into force, we estimate a fixed effects regression comparing years with a change and years where the reimbursement instrument remained the same. The model is adjusted for patient age and severity of the disease. The utilisation of DEB increased from 8,407 in 2010 to 19,065 in 2014. Hospitals used significantly more DEB when an additional payment instrument changed compared to years when it remained the same. The increase was roughly twice as large. In short, hospitals are incentivised to utilise new technologies if the reimbursement changes to an instrument that is designed in a more reliable way, e.g. including less bureaucracy or guaranteeing fixed prices.
\end{abstract}

\section{Keywords}

Additional payment instruments, diffusion of innovations, new health technology, hospital financing, DRG system, panel data

JEL

I12, I1 8, C32, C33 


\section{Introduction}

Every reimbursement system generates both adverse and beneficial incentives [1]. The design and structure of reimbursement systems is hence a major concern in health systems. With the aim to incentivise hospitals to provide care more efficiently, health policy-makers and insurers have moved away from fee-for-service to prospective payment systems and capitation [2]. Today, inpatient care in most OECD countries is financed with prospective payment systems, reimbursing services based on predetermined average expenses for diagnosis-related groups (DRG) [3]. Hospitals thus face the risk to be reimbursed less than their actual expenses per patient [4]. There is general consensus that DRG payments incentivise hospitals to utilise those technologies that lead to lower costs per patient [5-7]. Previous health economics literature has assessed in how far reimbursement, especially prospective payment models, influences the adoption and diffusion of new technologies [8-12]. Cappellaro et al. do not find evidence that higher levels of reimbursement encourage technology diffusion for the case of drug-eluting stents in different Italian regions [9]. Romeo and colleagues [11] analysed effects of reimbursement programmes with and without prospective payment on the diffusion of five capital-embodies technologies (e.g. electronic fetal monitoring). They show that prospective reimbursement influenced the diffusion of technologies considered, but effects depend on the specific attributes of prospective reimbursement policy and the technology itself. Even though consequences of prospective payment systems have been discussed extensively [13, 14], additional reimbursement instruments of DRG systems have not received much research attention: Prior studies based on cross-sectional designs [15] and surveys $[7,16]$ found that additional payment instruments may incentivise the utilisation of a new technology. SchellerKreinsen et al. [15] show that most of 12 investigated European countries used approaches of encouraging technological innovation [7]. Sorenson et al. [16] gave insides into the use of innovation payments of the National Health Service (NHS) in England from hospitals' perspective. However, only one third of surveyed NHS hospital finance managers $(n=20)$ believe that these payments effectively encourage the adoption of new technologies. Baeumler 
empirically examined factors that influence hospitals in their decision to use drug eluting stent in the treatment of acute myocardial infarction for patients of a large German sickness fund. Beside individual patient characteristics and hospital characteristics, the amount of additional payments was positively associated with the utilisation of drug eluting stents [15]. From a theoretical viewpoint, various studies have addressed the variation across hospitals adopting new technologies, while studies on individual hospitals adopting new technologies across time in the life cycle of a new technology is rather scarce. The incentive structure of additional reimbursement instruments for individual hospitals is, however, an essential aspect, as they are designed with the intention of balancing disincentives of DRG systems that might dominate decision-making authority [17-18].

The present longitudinal study analyses technology utilisation of hospitals over time across three different additional reimbursement instruments using fixed effects modelling. To our knowledge, this is the first study that examines the utilisation of new technologies in inpatient care while different reimbursement instruments are in place. We hypothesise that the utilisation of technologies increased abruptly when a new additional reimbursement instrument came into force. We study the effect by investigating hospitals' utilisaton of drug-eluting balloon catheters (DEB) between 2010 and 2014, while additional payment instruments changed twice within this time period.

\section{Background}

Studying the influence of incentives on human and organisational behaviour has become a core discipline in economic thought [19]. The initial idea in the theory of incentives is to recognise that contracts between principals and agents are linked to conflicting interests and unevenly distributed bargaining powers between the two parties, as Adam Smith observed first for wage contracts in agriculture $[20,21]$. Chester Barnard proposed that persuasion and tangible incentives are the relevant elements convincing subordinates in a system to cooperate. He defined four specific kinds of incentives, namely financial and material ones, non-material 
opportunities, physical work conditions and benefactions $[19,22]$. In the context of reimbursing inpatient care and especially when focussing on the diffusion of new technologies, both decision makers on policy and insurance level are acting as principals and (dis)incentivise hospitals to use new technologies. While the former is responsible for setting the frameworks for reimbursement, the latter is in power to negotiate additional payments. Reimbursement systems hence involve intended incentives that are implemented to change hospital behaviour, such as the introduction of DRG systems aimed to incentivise hospitals to act efficiently and effectively [1]. In addition, once implemented, reimbursement systems also involve unintended incentives in a way that hospitals in their role as agents use the system for their benefit [13]. Regarding the bundling of payments within DRG systems, there is general consensus that a disincentive exists for new technologies to be utilised when they are associated with increased costs per admission $[10,23]$. Many health care systems using DRGs thus maintain additional reimbursement instruments. The aim is to balance disincentives for hospitals to use new medical technologies while remaining in control of the decision whether to reimburse a particular technology [24].

The German DRG system bundles the expenses for infrastructure, services and materials. Their reimbursement rates are determined on the basis of diagnoses, procedures and patient characteristics such as age and gender [26]. Additional reimbursement instruments are installed for a technology when the incurred costs for a treatment vary substantially from standard payment rates. They are often used temporarily before a unique DRG is generated [7]. Varying in the particular design, previous literature has categorised additional reimbursement instruments as separate or supplementary payments: Separate payments are in most systems the first step to reimburse a new technology, sometimes before a procedure code has been assigned. They are negotiated locally or nationally at a time when information on the effectiveness of a technology is still relatively limited [25]. Supplementary payments are usually not limited to new technologies but include specific high-cost technologies to keep the DRG system more homogenous [7]. 
In the German inpatient reimbursement system, separate payments and two types of supplementary payments (supplementary payments with a negotiated price and supplementary payments with a nationally fixed price) are used. As the design of the additional payment instruments differs, the certainty and bureaucratic efforts for a hospital to receive a payment depend on the particular additional payment in place.

Separate payments for certain technologies are only eligible for hospitals that (1) requested permission to negotiate payments at the Institute of the Hospital Remuneration System (InEK), (2) got a positive decision for the technology in question from the InEK, and (3) were able to negotiate a payment amount with representatives of the payers. As the InEK's decision is valid for one year only, hospitals must request permission to negotiate payments at the InEK again for the next year if the technology in question fails to be included in the following year's lists of supplementary payments or the G-DRG classification. A previous study has identified that one third of all German hospitals was able to negotiate payments for one or more technologies eligible for separate payments [27].

Negotiated supplementary payments are determined by the InEk for certain technologies listed as an annex to the DRG catalogue. Opposed to additional payments, every hospital is allowed to negotiate a payment with the representatives of payers without prior request to the InEK. However, in the case of no agreement, insurers have to pay a lump sum of 600 euros per case. Neither the amounts of negotiated supplementary payments nor the negotiated amounts of separated payments are made public.

Lastly, fixed supplementary payments are the most reliable instrument for hospitals. The InEK sets a nationally fixed reimbursement price, i.e. all hospitals using the technology are eligible for remuneration without prior negotiation. While both kinds of supplementary payments are relevant for hospital budgets and volume thresholds will be negotiated, separate payments are, in theory, budget-neutral [28]. However, one should bear in mind that the negotiation of separate payments usually takes place within budget negotiations so that they nonetheless might 
have an indirect impact on the overall hospital budget even if volume thresholds for the technology are possible for separate payments. Table 1 outlines the main characteristics of German additional payments.

Tab 1 Additional reimbursement instruments in German inpatient care In conclusion, the change of reimbursement instruments implies a higher certainty for hospitals to receive (i) a reimbursement at all and (ii) a prospective determined amount of reimbursement. The first aspect occurs when changing from separate to negotiated supplementary payments and the latter one when changing from negotiated to fixed supplementary payments. Bureaucratic hurdles for additional payments thus decline continuously.

Based on the assumption that different additional payments instruments exert a varying degree of incentive for hospitals to adopt a technology, we selected a technology that was used by all additional payments systems consecutively for this study. Drug-eluting balloon catheters (DEB) are used in percutaneous transluminal coronary angioplasty (PTCA, in the following also referred to as "angioplasty") to treat coronary heart disease. After the first PTCA in 1977 [29] and its diffusion in the 1980s [30], bare-metal and then drug-eluting stents were developed to reduce frequent restenosis [31]. DEB have been developed since the mid 2000s and are considered to have advantages over drug delivery of stents due to their "broader area of surface contact and more homogenous drug-tissue transfer" [31]. One reason for selecting DEB are the sufficient number of angioplasty cases. Although age-standardised death rates had been decreased globally by 22 per cent between 1990 and 2013, cardiovascular and circulatory diseases are the primary cause of death in developed countries [32]. From an economic viewpoint, circulatory diseases account for more than 10 per cent of the health expenditures in OECD countries, which is the largest share of inpatient spending [33]. Most importantly, DEB are a suitable example to study the effects of additional reimbursement instruments as the technology was reimbursed in Germany by three different additional payments successively. In 2010, the first year of observation, DEB could be reimbursed as separate payments if a hospital had negotiated successfully. In 2011 and 2012, the reimbursement changed to negotiated 
supplementary payments. Hospitals either negotiated a payment or received a lump sum of $€$ 600 per utilised DEB. In 2013 and 2014, a nationwide fixed supplementary payment was set by the InEK. All private and public health insurances thus reimbursed this amount for a DEB utilisation. View the bottom part of Table 1 for further information of the reimbursement of DEB with additional payments across the years.

Reimbursement for DEB were paid in addition to DRG payments. These DRG payments were, for instance, 5,780 euros on average when performing a PTCA (F58) with heavy complications or co-morbidities (CC) in 2014 and 2,920 euros for a PTCA without CC. ${ }^{1}$ Slightly less frequent are PTCA with complex diagnosis (F52), yielding between 4,375 and 6,982 euros depending on CC and PTCA with highly complex intervention (F56) to be reimbursed with 3,756 to 7,016 euros. When using a DEB - being financed through fixed supplementary payments in this year - hospitals additionally received 836.37 euros.

\section{Methods}

\subsection{Data}

The study is based on a nationwide hospital data set called DRG statistic, provided by the Research Data Centers of the Federal Statistical Office and the statistical offices of the Länder. The DRG statistic contains patient-level data comprising all treatments and patients in German hospitals. For the purpose of this study, all patients undergoing coronary angioplasty in a German hospital between 2010 and 2014 were extracted and aggregated at hospital level per year. According to the longitudinal study design, we investigate the technology adoption per hospital and year. It captures all inpatient cases from any health insurance in Germany, ensuring that there is no selection bias due to patient sampling.

Relevant hospital cases were identified through the German procedure classification (OPS). We selected all treatments with the procedure code for angioplasty at the heart and coronary

\footnotetext{
${ }^{1}$ DRG payment rates are calculated by multiplying the respective cost weight of a DRG with the national 2014 average base rate of 3,156.82 euros, which in reality differed among the 16 federal states ranging from 1,113 and 3,325 euros.
} 
circulation system (8-837.0), marking those with an additional procedure code for DEB. Since 2011, the number of DEB used in a patient treatment has led to variations in the last digit of the procedure code. In this study, we do not differentiate if a patient received one or several DEB within the treatment. According to our research question, we analyse the effects of changing reimbursement instruments on hospital level. The DRG statistic defines a hospital as the entity that is bound by a budget agreement. In cases where a chain or network of hospitals is registered as one institution (i.e., with just one institutional code), it is considered to be one hospital.

\subsection{Variables}

In order to capture the utilisation of DEB most accurately, we used absolute and relative outcome measures for technology adoption. Dependent variables were the number of DEB per hospital and year in Model 1 and the share of DEB utilisations from all angioplasties per hospital and year in Model 2:

(1) number of DEB utilisations ${ }_{\text {it }}$ $=$ number of patients with DEB in hospital $_{i}$ and year t $_{t}$

(2) share of DEB utilisations ${ }_{i t}$

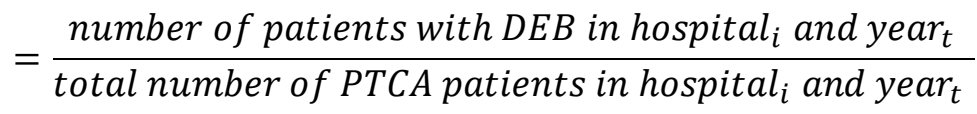

To account for the effect of additional reimbursement instruments in the respective years, we included dummy variables of the years of observation into our model [34]. This enables us to compare years with changing reimbursement instruments (2011 and 2013) and years when the reimbursement instrument remained the same (2010, 2012 and 2014). We hypothesise that the increase in utilisation is higher in years with a change in the reimbursement instrument than in years without.

Besides this main variable of interest, patient characteristics and the amount of reimbursement for the technology possibly influence the outcome and may vary over time. The explanatory variables as well as their hypothesised influence on the utilisation of DEB are listed in Table 2. 
In order to include them as covariates, we first tested their within variation to confirm that the variables changed over time.

Tab 2 Possible explanatory variables

Regarding patient characteristics, we consider the average age and mean severity of the patient cases per hospital and year. The data set includes patients undergoing PTCA between 40 and 90 years. Three observations of PTCA patients older than 90 years had to be excluded from the study due to reasons of data protection. To decrease multicollinearity among the predictor variables $[36,37]$, we standardised the age variable, rescaling it to have a mean of zero and a standard deviation of one. We also tested to use a second degree polynomial of the age variable to account for a possible non-linear relationship between age and the outcomes [38]. Based on the r-square test, the quadratic form did not lead to better explanatory power.

Besides that, the severity of a patient's disease may influence the decision to utilise a new technology $[10,39]$. The average severity across patient cases is measured by the case mix index (CMI), with many diffusion studies using the hospital or department CMI as covariate [9, 39]. It is calculated by the sum of the DRG relative weights divided by the number of cases in a hospital or department, representing the average resource consumption. When focusing on one specific technology, however, an adequate severity measurement for treated patients has to be developed, as done by Cutler and Huckman. They modified an index from Mark et al. [30] and divided angioplasty patients into three categories depending on the number of vessels blocked more than 70 per cent and a possible blockage of the artery or the left main trunk [10].

Compared to such medical measure, the CMI links medical severity to economic complexity. As procedures and their complexity are one essential part of determining DRGs and their relative weights, the case mix has an impact on how much a hospitals gets reimbursed for its services [40]. We thus calculated hospital- and year-specific CMI for angioplasty patients based on the DRGs used in the observed patient cases as a proxy for the average severity of patients undergoing PTCA. 


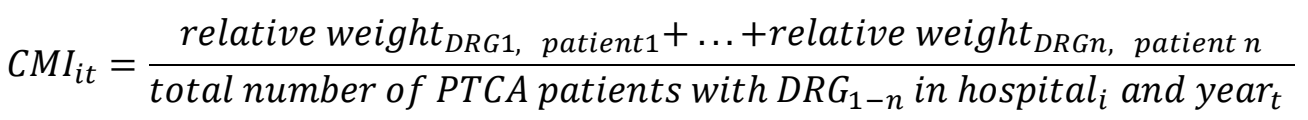

Lastly, the state-wide base rates determine the revenues from DRG payments to some extent, since the revenue is calculated by multiplying a DRG relative weight with the base rate [3]. Testing the within variation of the base rate suggested that the variable does not vary considerably between years. As it does not fulfil the criteria of time-variation for fixed effects regressions, we did not include this variable as covariate. Also the reimbursement amount of DEB could not be included as this information is only available for years of fixed supplementary payments.

\subsection{Econometric Model}

With the aim to assess the effect of changing reimbursement instruments on the utilisation of a new technology, we estimated a fixed effects regression, comparing the hospital's individual within variation before and after payment instruments changed. This approach is especially relevant for studying the adoption of new technologies since an extensive list of possible influencing factors exist that may lead to confounding [38]. Some factors are difficult to adequately control for, such as a patient's preference for a specific treatment and the physician's schools of thought. Since such factors influence a hospital in 2010 equally as in 2014, they do not bias the data estimation in the pre-post comparison of longitudinal research [41]. This is also the case for hospital characteristics such as number of beds per hospital, hospital ownership and university status. Keeping external factors constant, one can thus grasp longitudinal fixed effects regressions as approximation to an experimental logic [42]. Besides this contentual reasoning, we additionally conducted the Hausman test to ascertain that the fixed effects model was appropriate compared to a random effects model [41].

The regression can be written as the pooled model

$$
y_{i t}=\beta_{1} x_{i t}+\beta_{2} z_{i}+w_{i t} \quad w_{i t}=e_{i t}+u_{i}
$$


where $y_{i t}$ is the outcome under investigation, with the index it indicating that $y$ is subject to hospital-related and time-related explaining factors. $\beta_{1}$ is the estimated effect (coefficient) of time-variant factor $x_{i t}$ and $\beta_{2}$ is the estimation of the time-invariant factor $z_{i}$, taking on the same value for each time point. The error term $w_{i t}$ represents two parts, with $u_{i}$ being the cumulated effects of non-observed time-invariant factors and $e_{i t}$ the deviation of individual measurements from the individual specific measurement series (idiosyncratic error) [42]. Panel data for fixed effects regressions are transformed by subtracting the mean [41], so that the values of the time-demeaned data do not refer to an absolute height but to the deviation of the unit-specific mean:

$$
\left(y_{i t}-\bar{y}_{l}\right)=\beta_{1}\left(x_{i t}-\bar{y}_{l}\right)+\beta_{2}\left(z_{i}-z_{i}\right)+w_{i t} \quad w_{i t}=\left(e_{i t}+u_{i}\right)-\left(\bar{e}_{l}+u_{i}\right)
$$

After the transformation, the linear regression (Model 1) is performed with ordinary least squares, the regression of the relative model (Model 2) is performed with a logit link.

To correct for serial correlation and heteroskedasticity in the data set, we estimated the robust standard errors of the models [43]. The reported explanatory power (r-square) is taken from the standard linear regression instead of the fixed effects one, since the explanatory effects of the intercepts are removed in the estimation of the explanatory power in a fixed effect regression $[44,45]$. The likelihood ratio test was used to test the significance of model extensions and possible interactions. In order to compare the year coefficients with each other, we estimate the marginal effects $m_{i}$ of these variables [35]. The marginal effects represent the predicted means of DEB for each year. This also allows a direct contrast of the coefficients with measurements of significance. We used Stata 12.1 to estimate the regression models.

\section{Results}

The data set comprises 1,269,224 inpatient hospital admissions where a patient underwent PTCA in German hospitals between 2010 and 2014. Following the study purpose of analysing the effect of changing reimbursement instruments on the utilisation of DEB, the hospital 
admissions were aggregated per hospital and year. Across the years, on average six per cent of the balloon catheters were drug-eluting ones, corresponding to more than 72,000 patient cases where DEB were utilised. This relates to one hospital using a mean of 396 DEB in 2010 and a mean of 569 DEB in 2014. Table 3 gives descrives statistics on the PTCA treatment performed in German hospitals between 2010 and 2014.

Tab 3 Descriptive statistics on PTCA treatment in German hospitals from 2010 to 2014

More than half of these were planned surgeries with referral from outpatient care and 40 per cent of the cases were emergencies. Less than 27 per cent of the patients treated with DEB were women, which is roughly the same share as for standard balloon catheters, i.e. non-eluting ones. The share of women is higher for older patients: while 16 percent of the patients aged $40-50$ are women, they represent 45 per cent of the patients aged 80-90.

The descriptive statistics reveal substantial differences between the increase of DEB utilisation rates and standard balloon catheters. The number of DEB utilisation increased from 8,407 in 2010 to 19,065 in 2014, corresponding to an average yearly increase of 31.7 per cent. Standard balloon catheters increased by 2.5 per cent per year in average. The data set comprises 892 hospitals that conducted angioplasties between 2010 and 2014. The number of individual hospitals utilising DEB increased from 396 in 2010 to 569 in 2014. Figure 1 shows the development of DEB utilisation over time: Graph 1a displays the total utilisation numbers of DEB for all hospitals included in the analysis per year and their relative increase from one year to the next. Graph $1 \mathrm{~b}$ shows the average share of DEB utilisation within all PTCA interventions for all hospitals included in analysis. Additionally, Graph 1c displays the increase in the number of hospitals using DEB over the years including the relative increase of hospital from one year to the next. Graph 1d shows the development of the average number of DEB used per hospital and its relative increase.

Fig 1 Descriptive statistics of drug-eluting balloons between 2010 and 2014 
The descriptives suggest that the utilisation of DEB was higher in those years when a new statutory reimbursement instrument came into force (year 2011 and 2013) compared to years where it remained the same (years 2010, 2012, 2014). The transition probabilities from one year to the next reveal that 95.9 per cent of hospitals ever using one ore more DEB remained utilising them in the next period. Of those hospitals not using DEB in one period, 67.5 per cent continued not utilising DEB the following year.

Table 4 provides the results from the panel regression for two endpoints: the number of DEB utilisations (Model 1) and the share of DEB utilisations from all PTCA (Model 2) per hospital and year. The regression estimates show a positive time trend in all regression models. The probability of coronary angioplasty patients treated with DEB was 80 per cent higher in 2011 than in 2010 (logit = 1.39), significantly increasing from one year to the following (Model 2). The coefficient increased equally for the utilisation quantity (Model 1).

Tab 4 Regression estimates with robust standard errors and 95\% confidence intervals The predicted margins for the year variable indicate that a hospital used, on average, 11.6 DEB in 2010, 18.2 in 2011, increasing up to 28.0 in 2014, as displayed in Table 5. All predictive margins are significant at the 0.001 level in both models. Several aspects indicate that the increasing utilisation of DEB per year differs depending on a change of the reimbursement instrument. First of all, the 95\% confidence intervals of the coefficient for 2011 overlapped with the confidence intervals for 2012 in the fixed effects regressions, as it is the case for 2013 and 2014 (Table 4). In contrast, the confidence intervals are clearly distinct in those years when a change in reimbursement takes place. The overlapping confidence interval may indicate that the coefficients are not significantly different, which we, however, cannot conclude based on this [45]. To compare the coefficients between the years, we thus estimated the contrasts of the marginal linear predictions, as displayed in the lower section of Table 5.

Tab 5 Predictive margins and contrasts of marginal linear predictions of the year-covariate 
The same hospital used on average 6.63 DEB more in 2011 than in 2010, followed by 2.66 DEB more in 2012 versus 2011. Compared to the large difference in the first year of observation and the declined difference in the second, the difference increases again for 2013 versus 2012, when a new reimbursement instrument was introduced. It drops again for the following period when the reimbursement remained the same. The difference in the utilisation of DEB between 2010 and 2011 and between 2012 and 2013 was much larger than in the other years of observation. The effect decreased over time. The first period with a change of the reimbursement was larger than the second (6.63 compared with 4.94) and equally the first period without changing reimbursement was larger than the second (2.66 compared to 2.16). From a transitional perspective, 95.5 per cent of the hospitals ever using DEB remained using the technolgy in the next period. For those who did not use DEB in one period, 67.5 per cent remain not using them in the next.

We adjusted the model for the average severity of the disease and the average age of those treated with a DEB in the respective hospital and year. The association between the case mix index of the treated patients and a hospital's utilisation of DEB is negative and significant at the 0.05 level. This suggests that the number of patients treated with DEB is lower in hospitals with a higher case mix index, meaning that DEB were rather utilised in hospitals with a lower average severity of patients.

Overall, 85 per cent of the variance of the number of DEB can be explained by the covariates in Model 1 and 43 per cent of the variance of the share of DEB from all PTCA can be explained by the covariates in Model 2.

\section{Discussion}

Most OECD countries use additional reimbursement instruments to finance new health technologies in DRG systems, varying in the design and detailed structure of the mechanisms in place $[7,25]$. This study analysed the effects of changing reimbursement instruments on the utilisation of new health technologies. We focused on the variation across time, revealing how a 
hospital uses a new technology when additional reimbursement instruments change. Based on data of all patient cases treated with coronary angioplasty in German hospitals between 2010 and 2014, we found that changes in the additional reimbursement instruments cause a larger increase in the utilisation of drug-eluting balloon catheters (DEB) than in years when the payment instrument remains the same. We conducted several robustness checks within the framework of this study and this result was robust to each and every analysis conducted.

This observation suggests that the design of payment instruments generates incentives for technology adoption of hospitals. The more predictable and less beaurocratic it is to receive the payments, the larger the incentive to use a technology. The incremental increase in the use enables hospitals to gain more experiences with the new technology before utilising it in a broader scale.

The clear results were to some extent unexpected, since payment instruments are not primarily aimed at providing incentives but instead at reimbursing the new technology adequately for a hospital to utilise a new technology cost-neutrally. However, evidence on safety and effectiveness is in general the most important point in utilising technologies. Although stricter approval regulations for medical devices at EU level have been implemented until mid 2020 (Regulation (EU) 2017/745 of the European Parliament and of the Council of 5 April 2017), there is still a lack of evidence in routine care in the German health system. Generating evidence, especially in the early product livecycle, should be included in decisions and therefore in the design of payment instruments. Separate payments for certain high risk technologies have been under such framework of coverage with evidence development since a policy change in 2016. Having observed that the design of financial payment instruments is associated with the utilisation of technologies, generating additional evidence might be an useful design element also for supplementary payments, that provides further decision tools (1) for hospitals regarding the utilisation of technologies as well as (2) for payers regarding coverage and payment decisions. 
Besides that, it should be examined whether the results from the study can be applied to other technologies. DEB were a step-by-step development in a previously developed procedure. It thus does not involve a learning process as for an entirely new surgical procedure, so that no additional resources of physicians and hospitals beyond the technology cost are needed. Our results may be transferable to similar technologies moving through the different additional reimbursement instruments. The observed effects might differ when studying the utilisation of so-called "disruptive technologies", i. e. when no previous similar procedures or products exist [47]. We would expect that the diffusion would then be more focused on few early adopters among physicians.

Studying the technology utilisation of hospitals in a pre-post-comparison, we do not assess what institutional or external characteristics increase the likelihood of technology adoption. A previous study showed that being a larger hospital, a university hospital as well as being located in an area with a high degree of competition increases the chance for a hospital to negotiate additional payments with health insurances [27]. As this study is based on Germany-wide hospitals, it is plausible that the hospitals adopting DEBs in our study share these characteristics.

Concerning methodological assumptions for a causality of the results, the strict exogeneity assumption is fulfilled if the idiosyncratic error is uncorrelated with each explanatory variable across all time periods [48]. This assumption is relaxed to some extent in fixed effects models, since only time-variant unobserved factors need to be considered. Possible unobserved factors that coincide with utilising DEB could be a growing evidence of the effectiveness of DEB and the availability of the technology [49]. In fact, the European guideline for myocardial revascularisation from 2010 suggests that DEB "should be considered for the treatment of instent restenosis" after prior treatment with bare metal stents. However, the recommendation was based on a low level of evidence and the class of recommendation showed that there was conflicting evidence [50]. By 2014 the level of evidence was considered to be class A 
("procedure is beneficial, useful, effective") and class I of recommendation, recommending the use of DEB "for the treatment of in-stent restenosis within bare metal stents or drug-eluting stents" [51]. However, the change of the level of evidence does not coincide with the change in reimbursement instruments: Technologies are usually covered as separate payments for around two or three years. A technology is often transferred to the system of supplementary payments or included in the DRG classification at some point. This depends on different criteria that have not been made public by the InEK. However, the change from a negotiated supplementary payment to a fixed supplementary payment requires a sufficient number of patients to receive DEB and allows only for minimal variation in calculated costs to determine a fixed supplementary payment. In other words, if the number of patients for which DEB has been used is too low or variations in the calculated costs are too great, negotiated supplementary payments are used instead [52]. Secondly, the panel-attrition bias is a challenge for causal effects in unbalanced data sets [34]. The reason why hospitals left the sample ("attrition") must be uncorrelated with the idiosyncratic error. In our case, hospitals appear in one year but not in another if they merged or closed down in the meantime. The financing of DEB, even when taking all additional reimbursement instruments together, is by far too small and insignificant from overall hospital bills to be correlated with a hospital merging or closing down. It is also not convincing that hospitals in the data set treated patients with coronary angioplasty in some years but none in others, since coronary angioplasty is one of the major treatments of cardiology departments. These considerations make it unlikely that the panel-attrition bias influences the results. In short, we designed the study carefully to eliminate threats to causality.

Nonetheless, this study is subject to certain limitations. First of all, hospitals may utilise a technology without receiving a payment, which would still appear in the data as we selected the observation based on the procedure code. Due to cost pressures in the DRG system, this possibility can rather be excluded in reality. A frequent challenge of studies based on billing data is possible upcoding of DRGs due to financial incentives [53]. While there may be some hospitals doing more upcoding than others especially in the field of patient severity, this effect 
is mostly time-invariant and thus probably does not influence the results of the study to a relevant extent. Also, we probably underestimate the number of DEB in the final year of observation: The DRG data defines years by date of the hospital discharge. Since our last data year is 2014, patients who were hospitalised and treated in 2014 but discharged in 2015 do not appear in our data. This is, however, a limited effect given the short length of stay of PTCA and refers to about 1.3 per cent of the patients. Finally, a threat to diffusion studies is the prevalence of right censoring [6], meaning that non-adopters are included in the study who are not in the position to utilise the new technology. This would be the case for hospitals that do not make the decision between the old and the new technology in the first place, for instance because they do not meet some legal requirement for utilisation of the new one. Since the new and old technology in this study are used in the same procedure and in the same way, we do not see any reason why right-censoring should have occurred. Thus, using rigorous econometric analysis of longitudinal nationwide data, this study presents strong evidence that additional payment instruments exert incentives to utilise technologies.

\section{Conclusion}

The results of the present study substantially contribute to research on the diffusion of medical technologies by investigating the effect of changing additional payments on the utilisation of new technologies with higher costs than standard DRG rates in inpatient care. Using longitudinal nationwide data on hospital level, the analysis of the utilisation of drug-eluting balloon catheters (DEB) in the treatment of coronary heart disease shows that additional reimbursement instruments cause an increase in technology utilisation. The study confirms that additional payment instruments are generally embraced by hospitals and are appropriate to regulate technology diffusion, while they exert incentives themselves. In particular the different designs and intentions of the additional payment instruments thus have an impact on the utilisation of technologies. Since these incentives are of bureaucratic and financial nature, they may not be in line with medical decision making. At early times of technology diffusion with a 
high level of uncertainty regarding adverse events and the effectiveness of technologies, health systems have to consider approaches to generate evidence while reimbursing technologies by health insurances to balance the financial incentives. 


\section{REFERENCES}

1. Barnum, H., Kutzin, J., Saxenian, H.: Incentives and provider payment methods. Int J Health Plann Manage 10(1), 23-45 (1995)

2. McCullough, J.S.: The adoption of hospital information systems. Health Econ 17(5), 649664 (2008)

3. Pirson, M., Schenker, L., Martins, D., Dung, D., Chalé, J.J., Lecrercq, P.: What can we learn from international comparisons of costs by DRG? Eur J Health Econ 14(1), 67-73 (2013)

4. Hernandez, J., Machacz, S.F., Robinson, J.C.: US hospital payment adjustments for innovative technology lag behind those in Germany, France, and Japan. Health Aff (Millwood) 34(2), 261-270 (2015)

5. Kesteloot, K., Voet, N.: Incentives for cooperation in quality improvement among hospitals? The impact of the reimbursement system. J Health Econ 17(6), 701-728 (1998)

6. Lee, R.H., Waldman, D.M.: The diffusion of innovations in hospitals. Some econometric considerations. J Health Econ 4(4), 373-380 (1985)

7. Scheller-Kreinsen, D., Quentin, W., Busse, R.: DRG-based hospital payment systems and technological innovation in 12 European countries. Value Health 14(8), 1166-1172 (2011)

8. Bech, M., Christiansen, T., Dunham, K., Lauridsen, J., Lyttkens, C.H., McDonald, K., McGuire, A.: The influence of economic incentives and regulatory factors on the adoption of treatment technologies: a case study of technologies used to treat heart attacks. Health Econ 18(10), 1114-1132 (2009)

9. Cappellaro, G., Ghislandi, S., Anessi-Pessina, E.: Diffusion of medical technology: the role of financing. Health Policy 100(1), 51-59 (2011)

10.Cutler, D.M., Huckman, R.S. Technological development and medical productivity: the diffusion of angioplasty in New York State. J Health Econ 22(2), 187-217 (2003)

11.Romeo, A.A., Wagner, J.L., Lee, R.H.: Prospective reimbursement and the diffusion of new technologies in hospitals. J Health Econ 3(1), 1-24 (1984)

12.Sloan, F.A., Valvona, J., Perrin, J.M., Adamache, K.W.: Diffusion of surgical technology. An exploratory study. J Health Econ 5(1), 31-61 (1986)

13.Cots, F., Chiarello, P., Salvador, X., Castells, X., Quentin, W.: DRG-based hospital payment: intended and unintended consequences. In: Busse, R., Geissler, A., Quentin, W., Wiley, M. (eds): Diagnosis-related groups in Europe. Moving towards transparency, efficiency and quality in hospitals. Open University Press, Maidenhead, pp 75-92 (2011)

14.Farrar, S., Yi, D., Sutton, M., Chalkley, M., Sussex, J., Scott, A.: Has payment by results affected the way that English hospitals provide care? Difference-in-differences analysis. BMJ 339(7720), 554-556 (2009)

15.Bäumler, M.: Which non-clinical factors influence the use of innovative implants? The example of drug-releasing coronary stents in patients with acute myocardial infarction: a multilevel regression analysis. Das Gesundheitswesen 75(12), 822-831 (2013)

16. Sorenson, C., Drummond, M., Wilkinson, G.: Use of innovation payments to encourage the adoption of new medical technologies in the English NHS. Health Policy Technol 2(3), 168-173 (2013)

17.Busse, R., Ganten, D., Huster, S., Reinhardt. ER, Suttorp, N., Wiesing, U.: Zum Verhältnis von Medizin und Ökonomie im deutschen Gesundheitssystem: 8 Thesen zur Weiterentwicklung zum Wohle der Patienten und der Gesellschaft. Deutsche Akademie der Naturforscher Leopoldina e.V. 
https://www.leopoldina.org/uploads/tx_leopublication/Leo_Diskussion_Medizin_und_Oek onomie_2016.pdf (2016). Accessed 08 June 2018

18.Weiner, S.L., Maxwell, J.H., Sapolsky, H.M., Dunn, D.L., Hsiao, W.C.: Economic incentives and organizational realities: managing hospitals under DRGs. Milbank Q 65(4), 463-487 (1987)

19.Laffont, J.J., Martimort, D.: The theory of incentives: the principal-agent model. Princeton University Press, Princeton (2002)

20.Hall, B.H., Khan, B.: Adoption of New Technology. NBER Working Paper No. 9730. https://www.nber.org/papers/w9730.pdf (2003). Accessed 08 June 2018

21.Smith, A.: The wealth of nations. The Modern Library, New York (1776)

22.Barnard, C.I., Andrews, K.R.: The functions of the executive. Harvard University Press, Cambridge (1938)

23.Baker, L.: Managed care and technology adoption in health care: evidence from magnetic resonance imaging. J Health Econ 20(3), 395-421 (2000)

24.Clyde, A.T., Bockstedt, L., Farkas, J.A., Jackson, C.: Experience with Medicare's new technology add-on payment program. Health Aff (Millwood) 27(6), 1632-1641 (2008)

25.Sorenson, C., Drummond, M., Torbica, A., Callea, G., Mateus, C.: The role of hospital payments in the adoption of new medical technologies: an international survey of current practice. Health Econ Policy Law 10(2), 133-159 (2015)

26.Schreyögg, J., Stargardt, T., Tiemann, O., Busse, R.: Methods to determine reimbursement rates for diagnosis related groups (DRG): a comparison of nine European countries. Health Care Manag Sci 9(3), 215-223 (2006)

27.Ex, P., Vogt, V., Busse, R., Henschke, C.: The Reimbursement of New Medical Technologies in the German inpatient sector: What factors explain which hospitals receive innovation payments? HealthEcon, Pol, Law (2019)

28.Henschke, C., Bäumler, M., Weid, S., Gaskins, M., Busse, R.: Extrabudgetary ('NUB') payments: a gateway for introducing new medical devices into the German inpatient reimbursement system? J Manag Marketing Healthc 3(2), 119-133 (2010)

29. Gruntzig, A.: Transluminal dilatation of coronary-artery stenosis. Lancet 1(8058), 263 (1978)

30.Mark, D.B., Nelson, C.L., Califf, R.M., Harrell, F.E., Lee, K.L., Jones, R.H., Fortin, D.F., Stack, R.S., Glower, D.D., Smith, L.R. et al: Continuing evolution of therapy for coronary artery disease. Initial results from the era of coronary angioplasty. Circulation 89(5), 2015 2025 (1994)

31.Byrne, R.A., Joner, M., Alfonso, F., Kastrati, A.: Drug-coated balloon therapy in coronary and peripheral artery disease. Nat Rev Cardiol 11(1), 13-23 (2014)

32.GBD 2013 Mortality and Causes of Death Collaborators: Global, regional, and national agesex specific all-cause and cause-specific mortality for 240 causes of death, 1990-2013: a systematic analysis for the Global Burden of Disease Study 2013. Lancet 385(9963), 117$171(2015)$

33.OECD: Focus on Health Spending. Expenditure by disease, age and gender. http://www.oecd.org/els/health-systems/Expenditure-by-disease-age-and-gender-FOCUSApril2016.pdf (2016). Accessed 08 June 2018

34.Wooldridge, J.M.: Introductory Econometrics: A Modern Approach, 5th edn. South-Western Cengage Learning, Mason (Ohio) (2013) 
35.Mitchell, M.N.: Interpreting and visualizing regression models using Stata. Stata Press, College Station (2012)

36.Afshartous, D., Preston, R.: Key results of interaction models with centering. J Stat Educ 19(3) (2011).

37.Robinson, C., Randall, S.: Interaction effect. Centering, variance inflation factor, and interpretation issues. Multiple Linear Regression Viewpoints 35(1), 6-11 (2009)

38. Rabe-Hesketh, S., Skrondal. A.: Multilevel and longitudinal modeling using stata, 3rd edn. Stata Press, College Station (2012)

39.Adler-Milstein, J., Everson, J., Lee, S.Y.: EHR adoption and hospital performance: timerelated effects. Health Serv Res 50(6), 1751-1771 (2015)

40.Mabotuwana, T., Hall, C.S., Flacke, S., Thomas, S., Wald, C.: Inpatient complexity in radiology-a practical application of the case mix index metric. J Digit Imaging 30(3), 301308 (2017)

41.Cameron, A.C., Trivedi, P.K.: Microeconometrics using Stata, revised edn. Stata Press, College Station (2010)

42.Giesselmann, M., Windzio, M.: Regressionsmodelle zur Analyse von Paneldaten. VS Verlag für Sozialwissenschaften, Wiesbaden (2012)

43. Wooldridge, J.M.: Testing heteroskedasticity and autocorrelation in a fixed effects panel regression. https://www.stata.com/statalist/archive/2011-03/ms g00089.html (2011). Accessed 08 June 2018

44.Gould, W.: R-squared in panel data models. https://www.stata.com/statalist/archive/200305/msg00336.html (2003). Accessed 08 June 2018

45.Torres-Reyna, O.: Panel Data Analysis. Fixed and Random Effects using Stata (2007). https://www.princeton.edu/ otorres/Panel101.pdf. Accessed 08 June 2018

46.Knol, M.J., Pestman, W.R., Grobbee, D.E.: The (mis)use of overlap of confidence intervals to assess effect modification. Eur J Epidemiol 26(4), 253-254 (2011)

47.Jönsson, B.: Disruptive innovation and EU health policy. Eur J Health Econ 18(3), 269-272 (2017)

48.Brüderl, J.: Applied Panel Data Analysis. Using Stata. http://www.ls3.soziologie.unimuenchen.de/studium-lehre/archiv/teaching-marterials/panelanalysis-bruederl.pdf (2015). Accessed 08 June 2018

49.Lamiraud, K., Lhuillery, S.: Endogenous technology adoption and medical costs. Health Econ 25(9), 1123-1147 (2016)

50.Wijns W.: ESC/EACTS Guidelines on myocardial revascularization. European Heart Journal 31, 2501-2555 (2010)

51. Windecker S.: ESC/EACTS Guidelines on myocardinal revascularization. European Heart Journal 25, 2541-2619 (2014)

52.Henschke C., Bäumler M., Weid S., Busse R.: Extrabudgetary ('NUB') payments: A gateway for introducing new medical devices into the German inpatient reimbursement system? Journal of Management \& Marketing in Healthcare 3(2), 119-133 (2010)

53.Dafny, L.S.: How do hospitals respond to price changes? Am Econ Rev 95(5), 1525-1547 (2005) 